\title{
THESES ABSTRACTS
}

Bruce T. Ballinger, Complete extensions of ordered sets, McGill University, June 1969. (Supervisor: J. Lambek)

A comparison is made between the completions of ordered sets studied by Banaschewski [Hüllensysteme and Erweiterungen von Quasi-Ordnungen, Zeitschr. f. Math. Logik und Grundlagen d. Math. 2 (1956) 117-130] and the completions of categories studied by Lambek [Math. Lecture Notes 24 (1966) Springer Verlag]. However, in considering the completions of an ordered set, regarded as a category, functors into the category of sets are replaced by functors into the category 2. In particular, the following results are obtained.

The category of functors from $E^{O p p}$ to 2 is isomorphic to the largest closure extension of the (partially) ordered set $E$. The category of all those functors from $E^{o p p}$ to 2 which preserve all finite infima contains the ideal extension of Banaschewski; in fact, it is isomorphic to the latter when $E$ is a lattice. The category of all those functors from $E^{\text {opp }}$ to 2 which are products of representable functors is the Dedekind completion. It is observed that the Dedekind completion cannot be made into a functor in a natural way.

Robert Paré, Absolute properties in category theory, McGill University, June 1969. (Supervisor: J. Lambek)

A property preserved by all functors is called absolute. Absolute inverse limits are investigated; in particular a coequalizer is shown to be absolute if and only if a certain finite set of equations is satisfied. Absolute coequalizers are compared with Beck's contractible coequalizers; in fact Beck's "tripleableness" theorem is reformulated by replacing the latter concept by the former. This allows one to replace computational proofs involving systems of equations by conceptual proofs depending on the preservation of properties by functors. Also two new variants of Beck's "vulgar" tripleableness theorem are proved and illustrated.

Diagrams in additive categories which are preserved by all additive functors are called additively absolute. Conditions on direct limits to be additively absolute involve sums.

Properties of functors preserved by all hyperfunctors of the hypercategory of all categories into itself are also studied. Thus a functor between small categories preserves inverse limits absolutely if and only if it preserves monomorphisms absolutely. Assuming only that the source category has split idempotents, one may infer that such a functor has an adjoint.

K. L. Duggal, On a generalization of $\pi$-structures, University of Windsor, September 1969. (Supervisor: K. L. Eliopoulos)

The aim of the present work is to generalize the $\pi$-structures of G. Legrand (Thèse; Rendiconti del circolo Matematico di Palermo; Serie 2 , t. vii, 1958, pp. 323-354; t. viii, 1959, pp. 5-48) by considering 
a linear operator $J$ acting on the complexified tangent space $\mathrm{T}_{\mathrm{x}}^{\mathrm{C}}$ of a differentiable manifold $V_{n}$ satisfying a relation of the form $J^{r+1}=\lambda^{r+1}$ (identity), where $r \geq 1$ is an integer and $\lambda$ a nonzero complex constant. Such structures will be called Almost r-Product Structures, briefly, a.r.p.s.

We introduce the subject by giving the necessary historical background as well as some comments on important results.

We define an a.r.p.s. on a differentiable manifold $\mathrm{V}_{\mathrm{n}}$ (of class $\mathrm{C}^{\infty}$ ) and introduce bases adapted to this structure. This helps us to obtain a characterization of the infinitesimal connections (defined on the set of adapted bases which has a natural structure of principal fibre bundle) in terms of $\mathrm{J}$. Further, we generalize the concepts of curvature tensor and the holonomy group of these connections.

Next we consider a complex symmetric tensor $G$ on $V_{n}$ equipped with a.r.p.s. Introducing the compatability condition $J G=\lambda G$, we obtain a singular Riemannian structure subordinate to the a.r.p.s. By defining special adapted bases and special connections, we are able to get a characterization of these connections by conditions on $J$ and $G$. We also obtain a characterization of these singular Riemannian structures in terms of the holonomy groups of these connections.

In order to investigate the conditions for complete integrability of a.r.p.s.. we give a short introduction on completely integrable systems and construct a tensor determined on this structure which we call the torsion tensor.

The operators $\mathrm{C}$ and $\mathrm{M}$ of Lichnerowicz are generalized by the operators $\stackrel{\mathrm{S}}{\mathrm{C}}$ and $\stackrel{\mathrm{M}}{\mathrm{M}}$ as follows:

$$
\begin{aligned}
& \stackrel{\mathrm{s}}{\mathrm{C}} \phi\left(\mathrm{v}_{1}, \ldots, \mathrm{v}_{\mathrm{t}}\right)={ }_{\phi}\left(\mathrm{Jv}_{1}, \ldots, \mathrm{Jv}_{\mathrm{t}}\right) \\
& \stackrel{\mathrm{s}}{\mathrm{M}} \phi\left(\mathrm{v}_{1}, \ldots, \mathrm{v}_{\mathrm{t}}\right)=\sum_{\mathrm{k}=1}^{\mathrm{t}} \phi\left(\mathrm{v}_{1}, \ldots, \mathrm{Jv}_{\mathrm{k}}, \ldots, \mathrm{v}_{\mathrm{t}}\right)
\end{aligned}
$$

where $v_{1}, \ldots, v_{t} \in T_{x}^{c}, \phi$ is a $t$-form and $1 \leq \mathrm{s} \leq \mathrm{r}+1$.

The following are the main results on the study of these operators.

(a) Let $r$ be an odd integer, $s=\frac{r+1}{2}, \phi$ a linear form, and $T$ the torsion form of an a.r.p.s. Then,

$$
\lambda^{\mathrm{r}+1} \mathrm{~d} \phi+\stackrel{\mathrm{s}}{\mathrm{C}} \mathrm{d} \phi-\stackrel{\mathrm{s}}{\mathrm{M} d \stackrel{\mathrm{s}}{\mathrm{C}} \phi}=4 \lambda^{\mathrm{r}+1} \phi \cdot \mathrm{T} .
$$


Consideration of these operators $\stackrel{S}{M}, \stackrel{S}{C}$ also gives a local result for the torsion form $\mathrm{T}$ :

(b)

$$
T(u, v)=\frac{1}{-4 \lambda^{r+1}} \cdot \underset{s}{N}(u, v)
$$

where $T(u, v)=t_{j k}^{i} u^{j} v^{k} ; t_{j k}^{i}$ are the components of the torsion tensor and $\mathrm{N}(\mathrm{u}, \mathrm{v})$ is a generalization of the Nijenhuis tensor.

We again consider the complex symmetric tensor $G$ and say that $G$ is hermitian with respect to $J$ if

$$
\mathrm{JG}+{ }^{\mathrm{t}}(\mathrm{JG})=0
$$

where ${ }^{t}(J G)$ is the transpose of $J G$.

The resulting structure is called an almost $r$-product hermitian structure subordinate to the a.r.p.s., briefly H-structure. Such structures may exist on a differentiable manifold of a dimension which has to be a multiple of $(r+1)$ (where $r \geq 1$ is an integer). The manifold is not necessarily of even dimension as stated in the study of $\pi$-structures. Most of the other properties of the almost hermitian structures in the broad sense generalize in a natural way to the H-structures.

Finally, we examine some details which appear in the study of $\mathrm{H}$-structures by generalizing the concepts of hermitian and pseudo-hermitian structures, almost kählerian structures, kählerian and pseudo-kählerian structures. 